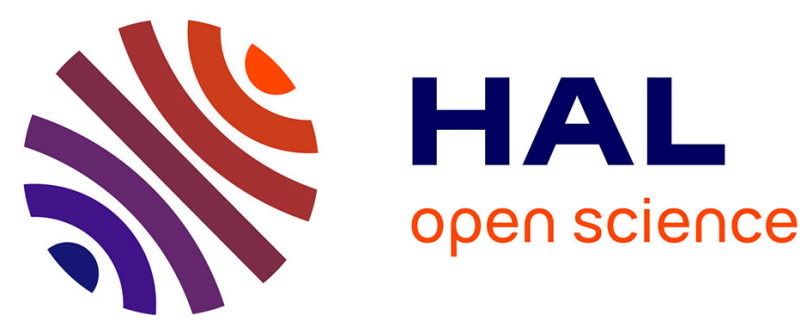

\title{
LA STRUCTURE DU COEUR DES DISLOCATIONS DANS LE SILICIUM CZ ETUDIEE PAR MICROSCOPIE ELECTRONIQUE
}

A. Bourret, J. Desseaux-Thibault, F. Lancon

\section{- To cite this version:}

A. Bourret, J. Desseaux-Thibault, F. Lancon. LA STRUCTURE DU COEUR DES DISLOCATIONS DANS LE SILICIUM CZ ETUDIEE PAR MICROSCOPIE ELECTRONIQUE. Journal de Physique Colloques, 1983, 44 (C4), pp.C4-15-C4-24. 10.1051/jphyscol:1983402 . jpa-00222841

\section{HAL Id: jpa-00222841 https://hal.science/jpa-00222841}

Submitted on 1 Jan 1983

HAL is a multi-disciplinary open access archive for the deposit and dissemination of scientific research documents, whether they are published or not. The documents may come from teaching and research institutions in France or abroad, or from public or private research centers.
L'archive ouverte pluridisciplinaire HAL, est destinée au dépôt et à la diffusion de documents scientifiques de niveau recherche, publiés ou non, émanant des établissements d'enseignement et de recherche français ou étrangers, des laboratoires publics ou privés. 


\title{
LA STRUCTURE DU COEUR DES DISLOCATIONS DANS LE SILICIUM CZ ETUDIEE PAR MICROSCOPIE ELECTRONIQUE
}

\author{
A. Bourret, J. Desseaux-Thibault et F. Lancon \\ Centre d'Etudes Nucléaires de Crenoble, Département de Recherche Fondamentale \\ et de Physique du Solide, $85 \mathrm{x}, 38041$ Grenoble Cedex, France
}

\begin{abstract}
Rêsumé - Les images de diverses dislocations <011> par microscopie électronique haute résolution de silicium $\mathrm{CZ}$ ont fourni des renseignements structuraux sur le coeur. Dans beaucoup de cas ces coeurs sont saturés par des impuretés oxygène qui vont jusqu'à former des cylindres de précipités sur certaines d'entre elles.
\end{abstract}

\begin{abstract}
Czochralski (CZ) silicon have been investigated by high resolution electron microscopy. Polycristals as well as deformed materials have been observed. The core structure of $60^{\circ}$ dissociated dislocation, Frank, Lomer, stair rod and [100]dislocations has been precised. The only pure and intrinsic structures are those of the $90^{\circ}$ Schockley partial. For al1 others oxygen impurity segregation is evidenced : this segregation may modify the core structure as for the Lomer stabilizing a configuration which is different from the Hörnstra model. The Frank and [100] dislocations contain a precipitate cylinder all along the $\langle 011\rangle$ core axis. The precipitate phase has been identified to be coesite, a high pressure $\mathrm{SiO}_{2}$ phase.
\end{abstract}

\section{I - INTRODUCTION}

La structure du coeur des dislocations dans les matériaux semiconducteurs de structure cubique diamant a fait 1 'objet de nombreuses recherches depuis quelques années : des résultats marquants ont été obtenus en utilisant la microscopie électronique haute résolution (MEHR). Ceux-ci ont porté sur le silicium $/ 1,2 /$, le germanium /3,4/ le CdTe /5/ et le CdS /6/. Ils ont montré que le coeur des dislocations était très peu. ëtendu sauf dans le cas d'une dissociation où apparâissent alors deux partielles en gënéral nettement séparées. L'intérêt actuel tient au fajt que le coeur d'une dislocation forme un système unidimensionnel très particulier : le réarrangement électronique qui accompagne les fortes distorsions et les défauts de coordinence peut se traduire par 1'apparîtion de 1iaisons reconstruites en chaîne 1inéaire /7/. Comme pour les spins magnétiques la notion de soliton le long de cette chaine de liaisons reconstruites a été introduite récemment pour expliquer les propriétés de charge et de dêplacement de ces dislocations /8/. Les reconstructions peuvent également se faire dans d'autres directions que la ligne de dislocation ; elles induisent alors des distorsions en cisaillement importantes /7/. Pour 1 'instant cependant aucune approche théorique self consistente n'existe : rēarrangement électronique et relaxation sont intimement 1 iés mais il est difficile de les introduire simultanément dans un calcul de bandes. Aussi; les seules tentatives de résolution des niveaux électroniques dus aux dislocations ont elles été faites en supposant la structure (donc T'emplacement des atomes) déjà connue /9/: Ta situation choisie ( $30^{\circ}$ non reconstruite) par Northrup et al. n'est d'ailleurs probablement pas 1 a meil leure puisqu'it $n^{\prime}$ 'est pas tenu compte d'une reconstruction. Tous les progrès théoriques actuels pour une meilleure évaluation des propriétés électriques des dislocations (densitê d'etat, structure de bandes) passent donc actuellement par une meilleure connaissance préalable de la structure.

Les propriétés de recombinaison, de luminescence et de frottement du rẻseau dêpendent de manière critique des détails du réarrangement atomique au niveau du coeur. Ces 
propriētés sont maintenant accessibles à 1 'expérience par cathodoluminescence $/ 10 /$, DLTS /11/, photoconduction /12/ ou EBIC /13/ sur des dislocations isolées et bien caractérisées.

Bien entendu, il subsiste cependant une ambiguité soulevée par Jaros et Kirton /14/. Les niveaux profonds détectēs par les mêthodes spectroscopiques sont-i is 1iês à $1 a$ structure intrinsèque des dislocations ou à des défauts (solitons, défauts ponctuels, décrochements gēométriques ou impuretēs) ? Cette question, en particulier la présence ou non d'impuretés, mérite qu'on s'y arrête et ne peut être élucidée que par une observation directe du coeur de ces dislocations.

Nous allons montrer en illustrant ceci sur plusieurs exemples de dislocations observées dans le silicium. les possibilitês de la MEHR et ses limites.

\section{II - LES DISLOCATIONS GLISSILES}

Les dislocations obtenues par déformation du silicium à haute température sont en général des dislocations à caractère vis ou des dislocations à $60^{\circ}$ alignées le long de direction $\langle 110\rangle$ [voir par exemple/15/]. Les premiēres observations en faisceau faible ont montré que celles-ci étaient dissociées en partielles. Les distances moyennes de dissociation sont très dispersées dans ce genre d'échantillion car les contraintes locales, surtout lors des déformations à relativement basse température, peuvent être importantes et modifient sensiblement les largeurs de dissociation. Il arrive même que certaines dislocations à $60^{\circ}$ soient non dissociēes. Par contre, dans les sous joints des polycristaux obtenus par tirage Czochralski (CZ) la dispersion des résultats est beaucoup moins grande.

La mesure directe en MEHR de ces largeurs de dissociation sur les dislocations à $60^{\circ}$ vues debout conduit à une valeur moyenne de $: d_{60^{\circ}}=5.4 \pm 5 \mathrm{~nm}$. Ceci donne une énergie de faute intrinsêque de $: \gamma_{s i}=62 \pm 7 \mathrm{~mJ} / \mathrm{m}^{2}$. Ces valeurs autorisent l'étude expērimentale séparēe des deux partielles différentes $30^{\circ}$ et $90^{\circ}$ résultant de la dissociation. (Les dislocations vis n'ont pas reçu une attention spéciale jusqu'à ce jour car elles contiennent deux partielles à $30^{\circ}$ ).

Plusieurs auteurs se sont attachés à résoudre la structure du coeur de la partielle à $30^{\circ} / 1,2,3 /$. Ils ont tous conclu à 7 'existence de la configuration "gliss $17 e^{\pi}$. Cependant des rêsultats plus récents /I6/ et une analyse plus détailiée des contrastes expérimentaux montrent que cette conclusion doit être nuancée. La distance mesurée entre les paires d'atomes $A$ et $B$ (Fig. 1) est de $1.6 \mathrm{~d}_{0}$ ( (où $\mathrm{d}_{0}=1 / 2<110>$ ), ce qui correspond trẽs bien aux valeurs calculées dans un modèle atomique par Marklund $/ 17 /$ en utilisant un potentiel de Keating aussi bien que la valeur de $154 \mathrm{~d}_{0}$ calculée par Masuda et Kojima $/ 18 /$ avec une approximation de liaisons fortes. Cependant, 1 'image de la colonne centrale $C$ du modẽle glissile $n$ 'est pas reproduite correctement par les images expérimentales, en particuijier quand les atomes sont imagés en bianc. De plus, la sensibi îté à la quantité exacte d'atomes dans la colonne centrale $C$ n'est pas grande, de sorte que les seules conclusions sưres que nous ayions actuellement sont les suivantes:

i) Il existe dans le coeur de la partielle $30^{\circ}$ un nombre d'atomes équivalent à $(1 \pm 0.5)$ colonne atomique. Ainsi, bien que la valeur moyenne corresponde à la configuration "glissile", il n'est pas possible d'exclure la présence d'une proportion relativement importante de défauts lacunaires ou interstitiels donnant localement à la dislocation une confiquration "shuffle"

ii) la colonne d'atomes située dans le coeur a un contraste très perturbé (noint d'intensité très fạble et allongé entre $A$ et $B$ pour une imaqe oú les atomes sont blancs). C'est ainsi que les imaqes simulées avec un modèle incluant une distribution de position dans le coeur entre $A$ et $B$, sont assez proches de 1 'expérience. Ceci veut dire, ou bien que la position de la colonne $C$ n'est pas bien définie (prếsence des défauts lacunaires ou interstitiels), ou bien que des impuretés yiennent ségréger dans cette partie sans toutefois construire un ordre bien défini. Cette seconde hypothèse parâ̂t la plus vraisemblable puisque toutes les autres dislocations de vecteur de Burgers plus important ont montré leur sensibilité aux impu-

rêtés oxygène. récsort que la présence de défauts (1acunes, interstitiels ou impuretés) est 
très probable dans la partielle à $30^{\circ}$. Cette conclusion vaut aussi bien dans les échantillons de Si CZ déformés que dans les dislocations de croissance de polycristaux CZ.

La dislocation partielle à $90^{\circ}$ a fait 1 'objet de très peu de travaux. Nos propres observations montrent que le contraste obtenu y est trẽs reproductible et correspond exactement à celui qui est simulé à partir du modẽle de Marklund $/ 17 /$. On doit noter qu'une éventuelle reconstruction des liaisons le long de 7 'axe <011> n'est pas détectable en $M E H_{3} R$ car les déplacements qui en rêsultent en projection sont très faibles $\left(2.5 \cdot 10^{-3} \mathrm{~nm}\right)$ et bien inférieurs à la résolution. Les observations en faisceaux faibles des dislocations à $60^{\circ}$ après déformation montrent que la partiel le à $90^{\circ} n^{\prime}$ 'est pas rigoureusement rectiligne et contient des décrochements : ceux-cj cependant sont assez difficiles à mettre en évidence lorsqu'on regarde la dislocation debout, car ils introduisent des variations de contraste très faibles quand leur nombre ne dépasse pas deux décrochements par $100 \AA$ de lame. Ainsi, la partịelle à $90^{\circ}$ contient peu ou pas de défauts, mais présente souvent des décrochements gēométriques simples (Fig. 2).

Ces observations sont en plein accord avec les résultats de Wessel et Alexander /15/ qui montrent que les partielles à $30^{\circ}$ sont beaucoup moins mobiles que les partielles à $90^{\circ}$. Les partielles à $30^{\circ}$ contenant des défauts dans leur coeur, la formation de doubles décrochements est plus difficile. A l'inverse, sur une partielle à $90^{\circ}, 1 e$ double décrochement est facile à former : sa vitesse, plus grande, est alors contrôlée par la propagation des simples décrochements qui peuvent être ralentis par des dēfauts extérieurs donnant ainsi l'apparence en zig-zag caractêristique de cette partielle.

\section{III - LES DISLOCATIONS SESSILES}

III-1. La dislocation de Lomer le long de $\langle 011\rangle$ et des plans de glissement (100) a été étudiêe en détail par Bourret et al. $/ 4 /$. Ce type de dislocation se rencontre aussi bien dans les sous joints qu'après déformation. Elle résulte de l'interaction: entre deux dislocations à $60^{\circ}$ de plan de glissement différent pour former ce qu'on appelle un blocage de Lomer-Cottrell: la configuration d'un tel blocage avait jusqu'à nos observations toujours été prévue comme étant dissociée en plusieurs partielles. Les dislocations d'interfaces des matêriaux III.V peuvent également réagir pour donner des dislocations de Lomer $/ 19 /$.

Les observations dans le silicium $\mathrm{CZ}$, comme dans le germanium, ont montré que cette dislocation n'était pas dissociée et avait une structure de coeur compacte. Une carte complète des positions atomiques autour du coeur a pu être dressée avec une résolution de l'ordre de $\pm 0.025 \mathrm{~nm}$. Comparées aux positions calculées par la théorie élastique, les positions ainsi déduites se révèlent différentes à l'intérieur d'ure zone de rayon $0.6 \mathrm{~nm}$, c'est à dire $\frac{3}{2} \mathrm{~d}_{\mathrm{o}}$. Cette zone que nous définissons comme étant celle du coeur contient 20 atomes par période le long. de la dislocation. La tendance générale des positions réelles est de respecter les longueurs des liaisons d'une meilleure façon que ne le laisserait prévoir la théorie élastique. L'image présente toujours une asymétrie caractēristique qui élimine le modēle simple contenant deux cycles d'atomes 5-7 superposés /20\%. Un modèle plus compliqué a donc été proposé, pour lequel 1 'accord entre images simulées et expérimentales est satisfaisant. Ce dernier contient une paire d'atomes en position de faute et des liaisons non saturées à reconstruire (Fig. 3 ).

La reconstruction introduit une énergie importante de cisaillement, aussi il est possible que la configuration asymétrique soit en fait stabilisée par des impuretés dont nous savons qu'elles affectent plus ou moins ces dislocations. Pour clarifier ce point, nous avons procédé à des calculs d'énergịe des diverses structures sur ordinateur.

La méthode employée pour les calcuis de simulation est relativement similaire à celle utilisée par Marklund $/ 17 /$. Elle consiste à déplacer les atomes de coeur de manière à minimiser leur énergie potentielle. Le potentiel interatomique utilisé est celui de Keating, qui dépend de deux paramètres, $\alpha$, le paramètre d'élongation de la liaison covalente, $\beta$, Te paramètre d'orientation angulaire de cette liaison. Les valeurs respectives de $\alpha$ et $\beta$ ont fait récemment 7 'objet de controverses, aussi avons nous choisi deux séries de valeurs. Les valeurs classiques de $\alpha=48.50 \mathrm{~N} / \mathrm{m}, \beta=13.81 \mathrm{~N} / \mathrm{m}$ données par MARTIN $/ 21 \%$ (potentiel I) et les valeurs plus récentes de $\alpha=51.51 \mathrm{~N} / \mathrm{m}$, 
$B=4.70 \mathrm{~N} / \mathrm{m}$ données par BARAFF et a1. $/ 22 /$ (potentiel II), qui tolèrent des distorsions angulaires beaucoup plus facilement que le potentiel $I$. Ce potentiel empirique est certes criticable : il n'est valable que pour de fajbles déformations et surtout 11 suppose que chaque atome reste tetracoordonnē. Les 1 jaisons pendantes non reconstruites sont ainsi difficiles à prendre en compte à moins d'introduire des atomes virtuels. Dans le cas d'une dislocation de Lomer cependant des reconstructions le long du coeur sont assez faciles à imaginer. Deux modèles ont été considérés : le modèle de Hörnstra (A)et le modèle à coeur asymétrique (B). Pour le modè e A 1a reconstruction estêvidente et donne les cycles 5-7 d'atomes. Pour le modèle $B$ la reconstruction peut se faire facilement entre les atomes $a$, b et $c, d$ avec deux configurations différentes : la configuration chaise (B1) et la configuration bateau (B2).

La figure 4 montre les configurations de coeur pour les modèles $A$ et B2 avant relaxation (positions données par la théorie élastique) et après relaxation avec le potentie III, les atomes périphëriques ayant été maintenu fixes. Les énergies correspondantes figurent sur le tableau 1. De cette étude, il ressort les points suivants : i) la configuration d'énergie la plus basse est celle de Hornsträ (A), quel que soit le potentiel utilisé. $i i)$ vient ensuite la configuration B2 bateau, d'énergie $70 \%$ plus élevêe. Pour cette configuration d'importants déplacements le long de $<011>$ ont lieu pour la paire d'atomes $b, c$, qui entrânent les atomes voisins. De plus, comne cela a été remarqué expérimentalement la relaxation conduit à rendre les longueurs des liaisons plus proches de la valeur normale du cristal parfait. La forme du cycle à 8 atomes est cependant plus circulaire sur le modèle calculé que sur l'image expérimentale : la distance entre les paires d-e est de $0.61 \mathrm{~nm}$ (exp) au lieu de $0.52 \mathrm{~nm}$ (calc). iji) la configuration B doit être stabilisée par d'autres facteurs teis que des impuretẽs. Il est en effet possible d'introduire des atomes d'oxygēne dans la partie du coeur correspondant aux reconstructions. Ceci permettrait un relachement des contraintes de cisaillement et pourrait stabiliser la structure asymétrique (B). Les différences de position qui subsistent sur les paires d et e pourraient s'expliquer par $l^{\prime}$ introduction d'atomes supplëmentaires d'oxyg̣êne qui tendrajent à écarter ces paires.

Ainsi 1 'inactivité électrique des dislocations de Lomer /19/ s'explique bien par le fait que les liaisons sont reconstruites ou saturées par des impuretés. De plus, l'énergie de coeur contrôle ici complètement la faculté qu'a une dislocation à se dissocier en blocage de Lomer cottrel1. Bien qu'élastiquement favorable cette dissociation ne se fait pas car l'énergie de coeur des trois partielles créées serait trop élevée.

III-2. La dislocation de Frank (Fig. 5) de vecteur de Burgers $1 / 3<111>$ est très souvent observée dans les sous-joints des polycristaux de Si CZ bruts de tirage. Elle est toujours associée à d'autres partielles pour former des configurations complexes associant 3 partielles de Frank ou des partielles de Schockley à $90^{\circ} / 23 /$ identiques à celles observées dans le germanium. On retrouve également cette dislocation dans les boucles fautēes produites après précipitation d'oxygène dans Si /24/ ou en bordure des fautes d'empilement extrinsèques dues à la croissance d'oxyde $/ 25 /$. C'est dire son importance pratique. On doit cependant distinguer les Frank bordant une faute intrinseque lacunaire (1) ou interstitielle (2) de celle bordant une faute extrinsěque interstitielle (3). Il existe également une Frank bordée d'une faute extrinsẽque d'un côté et d'une faute intrinsèque de 1 'autre (4). La Frank n'a jamais étê observêe pure : elle contient un cylindre de matière ségrégée que nous avons attribué à l'oxygène. La taille de ce cylindre dans toutes les dislocations de tirage est de l'ordre de $\mathrm{d}=0.7 \mathrm{~nm}$ et le coeur contient environ 4 atomes d'oxygẽne par période le long de $<011>/ 26 /$. La configuration hypothétique des dislocations de Frank pures contient des cycles 5-7 d'atomes avec reconstruction identique à une partielle $90^{\circ}$ sauf dans 1 e cas de la Frank (4) qui, très perturbée, doit être facile à attaquer et sur lequel 7 'oxygène doit pouvoir se fixer facilement. C'est en effet celle-ci qui prêsente $7 a$ décoration maximum $(d=0.9 \mathrm{~nm})$. Une fois amorcée la précinitation est favorisée par je relâchement de contrainte qu'elle entraîne /27/.

III-3. La dislocation $\frac{1}{3}[\overline{000]}$. Cette dislocation un peu particulière résulte de l'intersection de deux ${ }^{3}$ fautes d'empilement intrinsèques et provient par exemple de 1a dissociation suivante : (Fig. 6)

$\frac{1}{2}[\overline{2} \mid \bar{i}] \longrightarrow \frac{1}{3}[\overline{1} \bar{i}]+\frac{1}{6}[\overline{2}, \bar{i}]+\frac{1}{3}[\overline{100}]$ 
Elle présente un intērêt particulier au niveau structural car elle donne des images expérimentales très reproductibles et très simples. Il y a en particulier une remarquable symétrie par rapport au plan [200]. De plus, comme pour les partielles $30^{\circ}$ ou $90^{\circ}$, il est facile de tester si $1^{\prime}$ 'interprétation des images est correcte en regardant le contraste des fautes d'empilement adjacentes qui n'ont pas la même symétrie lorsque les atomes sont blancs ou noirs /1/. Les images expërimentales de ces observations comportent une partie oū l'emplacement des atomes n'est pas résolu. La structure observée étant très reproductible une forte stabilisation par réarrangement électronique ou absorption sélective d'impuretés doit avoir lieu sans que nous puissions actuellement la préciser.

III-4. La dislocation [100]. Ces dislocations n'existent que dans les sous joints $\left(1^{\circ}\right.$ à $\left.5^{\circ}\right)$. Elles se présentent dans le silicium sous plusieurs formes correspondant à la forme non dissociée et aux dissociations suivantes /28/ :

$$
\begin{aligned}
& {[\bar{i} 00] \longrightarrow \frac{1}{3}[\bar{i} i]+\frac{1}{3}[\overline{2} \mid \bar{i}]} \\
& \text { ou }[\bar{i} 00] \longrightarrow \frac{1}{6}[\overline{2} i \bar{i}]+\frac{1}{3}[\overline{i 00}]+\frac{1}{6}[\overline{2} \bar{i} 1]
\end{aligned}
$$

La forme non dissociēe $n$ 'est vraisemblablement pas stable puisqu'elle est toujours très dêcorée et contient un grand cylindre de matière sêgrégée : il s'agit d'une phase particulière haute pression de $\mathrm{SiO}_{2}$, la coesite. Le cylindre a une taille constante et bien reproductible de $d=2,0 \mathrm{~nm}$ et $i 1$ contient environ 40 atomes d'oxygène par période le long de $<011>$. L'identification de la phase coesite s'est fajte, d'une part, par analogie avec le cas du germanium qui présente des structures jsomorphes, d'autre part par simulation d'image en utilisant la projection le long de $\vec{a}$ de la structure coesite (BOURRET et al.) à paraître). Les formes dissociées par contre produisent des partielles dont les propriétés sont identiques aux partielles semblables observées sur les autres dislocations (structure et sensibilitê à l'oxygène).

\section{IV - CONCLUSION}

L'ẹtude des dislocations par MEHR a révélé qu'il était assez rare d'observer les structures intrinsèques de coeur. Seules les partielles à $90^{\circ}$ de Schockley semblent dénuées d'impuretés : les positions des atomes correspondent alors aux modèles atomiques. Dans la partielle à $30^{\circ}$ nous avons mis en évidence une colonne atomique assez perturbée par la présence de défauts (ponctuels ou impuretés?). Pour toutes les autres dislocations il n'a pas été possible d'observer des structures intrinsèques dans le silicium $\mathrm{CZ}$. Dans la dislocation de Lomer bien qu'assez faiblement décorée, une paire d'atomes en positions de faute est vraisemblablement stabịlisée par des impuretés. Les dislocations de Frank et [100] comportent dans un cristal brut de tirage un cylindre de matière précipitēe que nous avons identifié comme ètant 1 a coesite phase $\mathrm{SiO}_{2}$ haute pression. L'étude de silicium beaucoup plus pur (fusion de zone) serait sans doute prometteuse pour accéder aux structures de coeur intrinsèque. Cependant on s'éloignerait aussi des conditions pratiques d'utifisation du silicium. La prise en compte des effets des impuretês apparait ainsi comme primordiale pour tous les défauts de croissance. Quelques résultats préliminaires nous ont montré qu'il en est de même pour les défauts qui résultent de traitement thermique. Ainsi, il faut être particuliērement prudent pour interpréter les résultats des mesures êlectriques ou spectroscopiques car la présence d'impuretês peut très bien nodifier complètement les niveaux d'énergie profonds dus au coeur d'une dislocation intrinsèquement pure.

\section{REFERENCES}

1/ OLSEN A., SPENCE J.C.H., Phil Mag A 43(1981)945.

2/ ANSTIS G., HIRSCH P.B., HUMPHREYS C., HUTCHINSON J., OURMAZD A., Inst. Phys. Conf. Ser. 60(1981)15.

3/ BOURRET A., DESSEAUX j., D'ANTERROCHES C., Inst. Phys. Conf. Ser. 60(1981)9.

4/ BOURRET A., DESSEAUX J., RENAULT A., Phi] Mag A 45(1982)1.

5/ YAMASHITA T., PONCE F.A., PIROUZ P., SINCLAIR R., Phil Mag A 45(1982)693.

6/ ECHIGOYA J., PIROUZ P., EDINGTON J.W. A A $45(1982) 455$. 
7/ MARKLUND S., Phys. Stat. Sol. (b) 92(1979)83.

8/ HEGGIE M., JONES R., Phil Mag (à paraître).

9/ MORTHRUP J.E., COHEN M.L., CHELIKOHSKY J.R., SPENCE J., OLSEN A., Phys. Rev. B 24(1981)4623.

10/ PENNYCOOK S.J., BROWN L.!., CRAVEN A.J., Phil Mag A 41(1980)589.

11/ KIMERLING L.C., PATEL J.R., BENTON J.L,, FREELAND E., Inst. Phys. Conf. Ser. 59 (1981) 401 .

12/ MERGEL D., LABUSCH R., Phys. Stat. Sol(a) 69(1982)151.

13/ PASEMANN L., BLUMTRITT H., GLEICHMANN R., Phys. Stat. Sol(a) 70(1982)197.

14/ JAROS M., KIRTON M.J., Phil Mag 46 B(1982)85.

15/ WESSEL K., ALEXANDER H., Phi] Mag 35(1977)1523.

16/ BOURRET A., DESSEAUX-THIBAULT J., D'ANTERROCHES C., PENISSON J.M., DE CRECY A., J. of Microscopy $129(1983) 337$.

17/ MARKLUND S., Phys. Stat. Sol(b) 100(1980)77.

18/ MASUDA K., KOJIMA K., J. Phys. Soc. Jap. 51(1982)1510.

19/ PETROFF P.M., LOGAN R.A., SAVAGE A., J. MTCrosC. $118(1980) 256$.

20/ HORNSTRA J., Physics Chem. Solids 5(1958)129.

21) MARTIN R.M., Phys. Rev. B 1 (1970)4005.

22/ BARAFF G.A., KANE E.0., SCHLUTER M., Phys. Rev. 8 21(1980)5662.

23) BOURRET A., DESSEAUX-THIBAULT J., PhiT Mag A 39(1979)405.

24/ MAHER D.M., STANDINGER A., PATEL J.R., J. AppT. Phys. $47(1976) 3813$.

25/ LEROY B., J. App1. Phys. 53(1982)4779.

26/ BOURRET A., COLLIEX C., TREBBIA P., J. Physique Lettres $44(1983) \mathrm{L}-33$.

$27 /$ BOURRET A. (cette conférence).

28/ DESSEAUX-THIBAULT J. (cette conférence).

Tableau 1. Energie totale du coeur de la dislocation de Lomer calculée avant relaxation $\left(E_{0}\right)$ et après relaxation $\left(E_{R}\right)$. Cette énergie est donnée pour 69 atomes par période, $d_{0}$, le long de la dislocation.

\begin{tabular}{|c|c|c|}
\hline Configuration & Pot 1 /21/ & Pot 2 /22/ \\
\hline Symétrique (A) & $E_{R}=4.73 \mathrm{eV}\left(E_{0}=8.50 \mathrm{eV}\right)$ & $2.47 \mathrm{eV}\left(E_{0}=7.12 \mathrm{eV}\right)$ \\
Asymétrique B1 (chaise) & $10.76 \mathrm{eV}(57.44 \mathrm{eV})$ & $5.40 \mathrm{eV}(52.62 \mathrm{eV})$ \\
Asymétrique B2 (bateau) & $8.27 \mathrm{eV}(57.44 \mathrm{eV})$ & $4.19 \mathrm{eV}(52.62 \mathrm{eV})$ \\
\hline
\end{tabular}



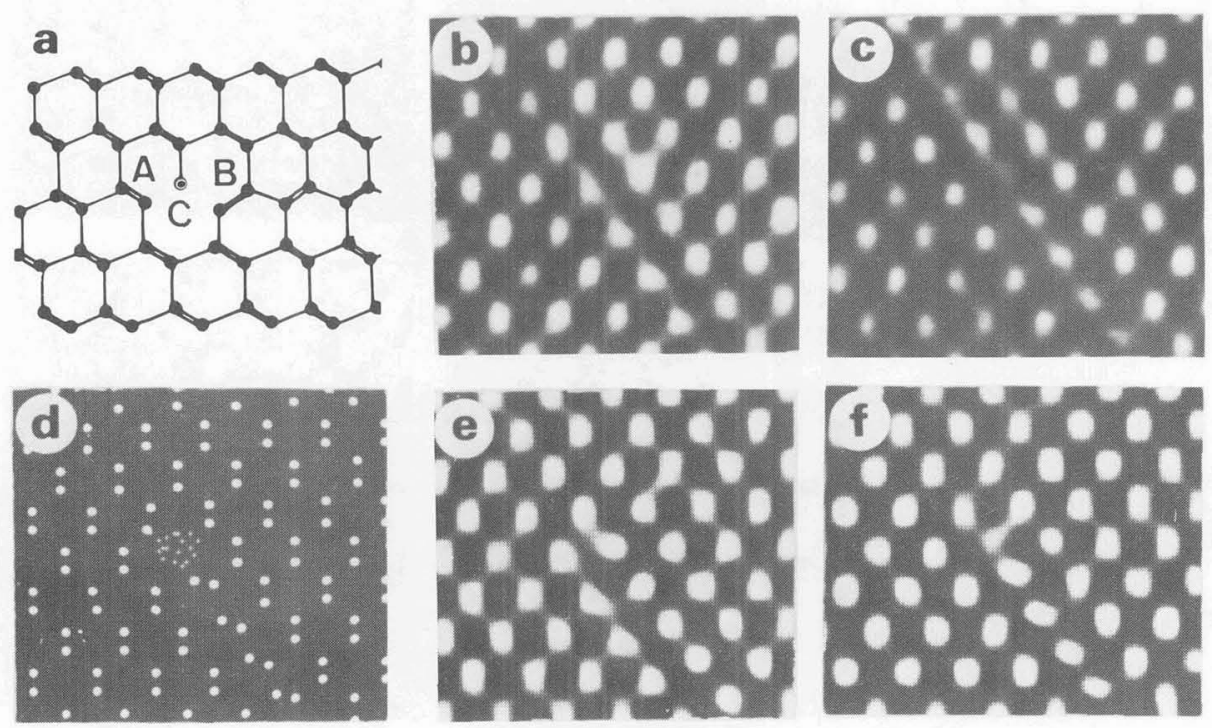

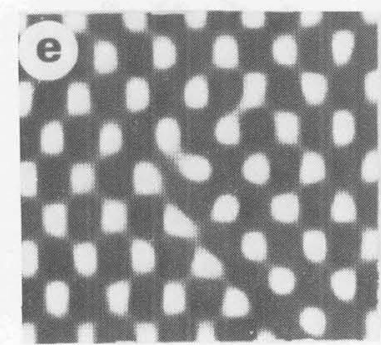

$\triangle Z=-50 \mathrm{~nm}$

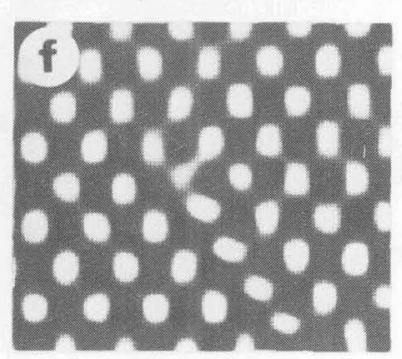

$\triangle Z=-90 \mathrm{~nm}$

Fig. 1 Partielle à $30^{\circ}$ projetée selon [011]. a/Modèle de la configuration glissile avec une colonne atomique en C. b/c/Images expérimentales à $200 \mathrm{keV}$ (résolution 0.25 $\mathrm{nm}$, êpaisseur $10 \mathrm{~nm}$ ) pour deux défocalisations avec atomes respectivement noirs et blancs. d/Modēle projeté qui donne le meilleur accord entre les images simulées c/ et $f /$ et les images expérimentales b/ et $c /$. La colonne $C$ se compose en fait d'atomes dont la position (et la nature) est mal définie.

$30^{\circ}$ partial dislocation projected along $[0 / 1]$. a/Glide configuration. $b / c /$ Experimental images using HREM at $200 \mathrm{keV}$; resolution $0.25 \mathrm{~nm}$; specimen thickness $10 \mathrm{~nm}$; two defocussing distances giving black or white atoms. The projected model $\mathrm{d} /$ fits the experimental images when compared to the simulated images e/ and $\mathrm{f} /$. The atomic column $C$ is in fact composed with randomly located atoms between pairs $A$ and $B$. 

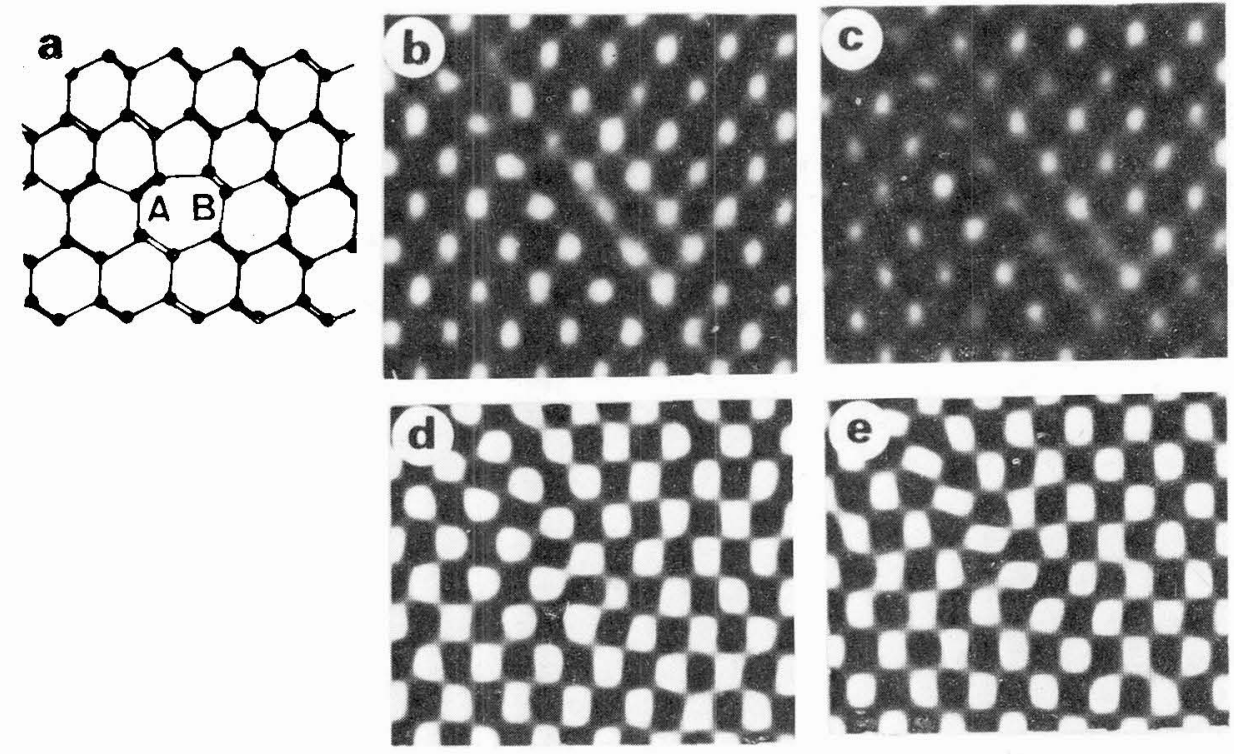

$-50 n m$

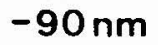

Fig. 2 Partielle ã $90^{\circ}$ projetēe selon [0/1]. a/Modèle de Marklund. b/ et c/Imag̣es expérimentales comparées aux images simulées d/ et e/ pour deux défocalisations donnant des atomes noirs ou blancs, $200 \mathrm{keV}$, épaisseur $\approx 8 \mathrm{~nm}$.

$90^{\circ}$ partial dislocation projected along [0/1]. a/ Marklund model. b/ and c/ Experimental images are compared with the simulated ones d/ and e/ at two defocussing distances giving black or white atoms - $200 \mathrm{keV}$ - specimen thickness $8 \mathrm{~nm}$.

A

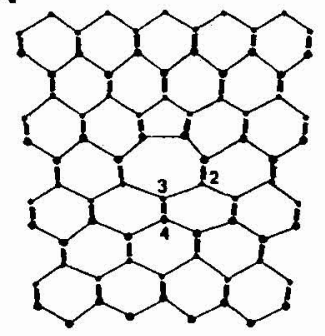

B

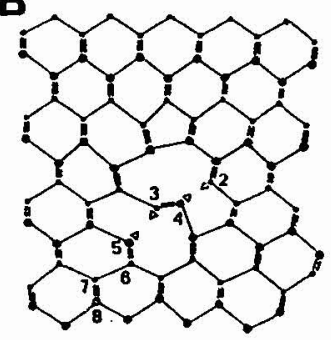

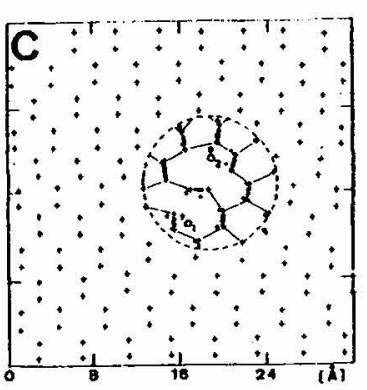

Fig. 3 Schêma des modèles atomiques de Hörnstra A/ et celui proposé par BouRRET et aT. B7 pour une dislocation de Lomer. Les positions atomiques déduites des images haute résolution expérimentales sont figurées en $\mathrm{C} /$ : elles se rapprochent du modèle $B /$.

Two different core models of the Lomer dislocation due to Hörnstra A/ and BOURRET et al. B/. The atomic positions as deduced from the HREM experimental images are reproduced at $\mathrm{C} /$. 


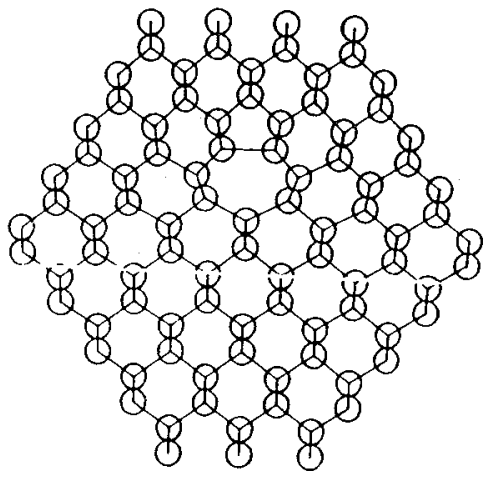

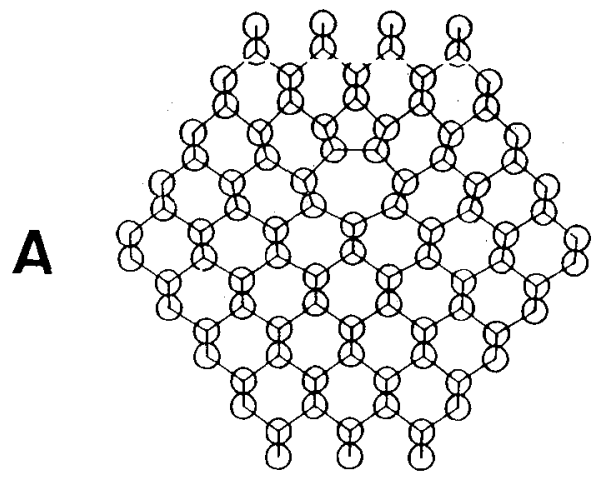

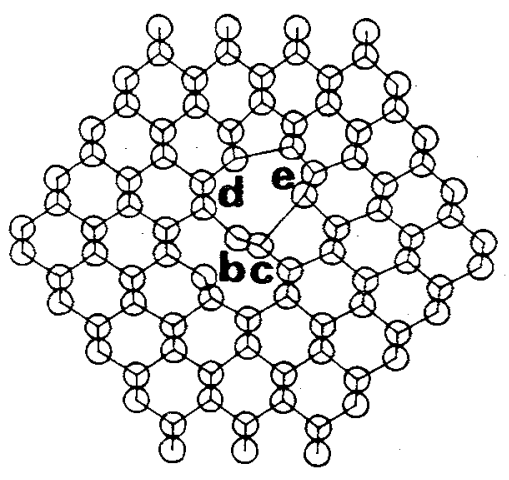

non relaxé

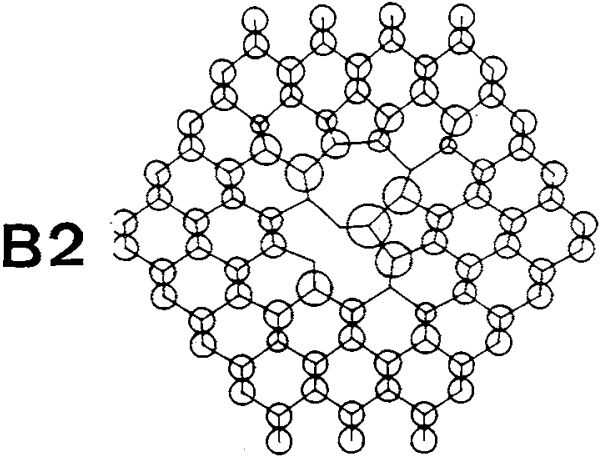

relaxé

Fig. 4 Configurations de coeur calculées en utilisant un potentiel de Keating pour Tes deux modèles $A$ et B2 de la dislocation de Lomer. Les schémas non relaxés correspondent aux positions initiales données par la théorie élastique. Les positions rela xées correspondent à 7 'état d'énergie potentielle minimale. La représentation estune section à 7 a cote $z=0.25 d_{0}$ qui coupe des sphères centrées sur chaque atome et ayant pour diamètre la distance interatomique. La relaxation le lon de l'axe de la dislocation <011> est apparente pour le modèle B2.

Calculated core configurationsof models $A$ and $B$ for the Lomer dislocation. Unrelaxed positions corresponds to elastically calculated atom location. The minimum energy configuration is the relaxed one. The representation is a section at $z=0.25 \mathrm{~d}$ 。 which cuts spheres centered at atoms with diameters equal to the interatomic distance. Relaxation along $\langle 011>$ is evidenced by circle radius variation for model B2. 

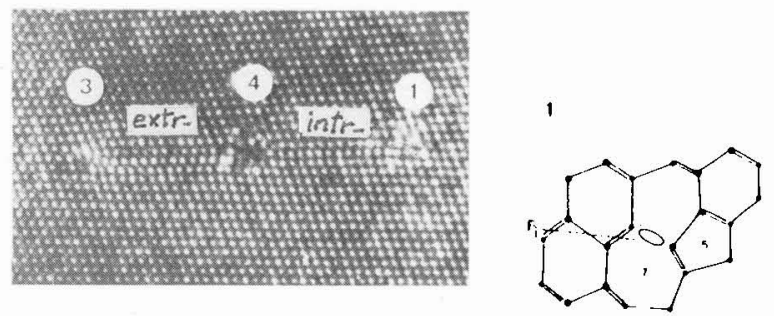

2
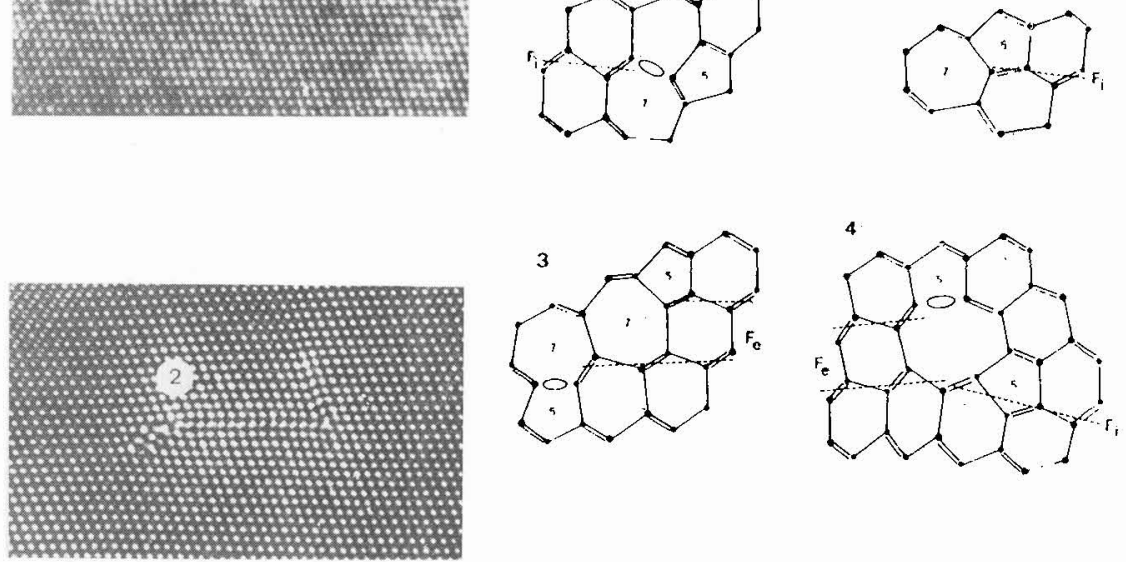

Fig. 5 La dislocation de Frank sous ses quatre formes observées expérimentalement.(1) bordant une faute intrinsèque lacunaire; (2)bordant une faute intrinsèque interstitielle; (3)bordant une faute intrinsèque interstitielle; (4)bordée par des fautes intrinsèque et extrinsèque. Les modèles proposés correspondent à des dislocations "pures".

The Frank dislocation four forms as observed experimentally. (1)vacancy intrinsic stacking fault; (2)interstitial intrinsic fault; (3) interstitial extrinsic fault; (4)between intrinsic and extrinsic faults. The proposed models correspond to dislacations.

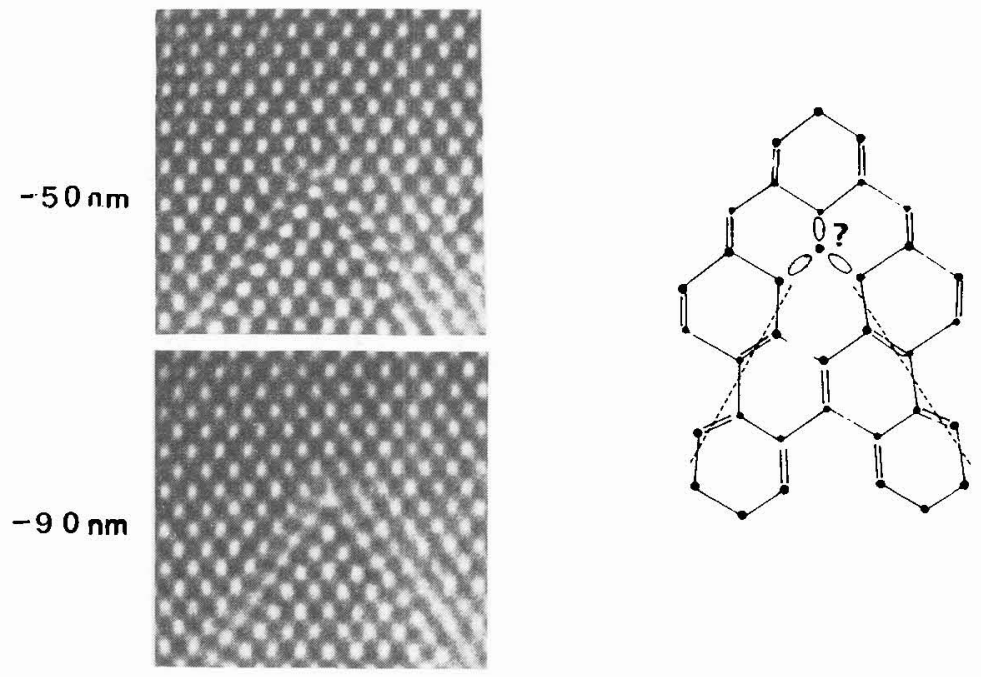

Fig. 6 La dislocation $1 / 3[100]$ vue à deux défocalisations différentes et 1'emplacement schëmatique des atomes. La partie centrale est difficile à préciser car les atomes n'y sont pas résolus.

The 1/3[100] dislocation observed at two defocussing distances and schematic location of atoms. The central part is difficult to precise as atoms are not resolved. 